\title{
A Smart Supervisory Control System Framework for a Sugar Mill Crystallisation Stage
}

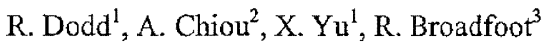

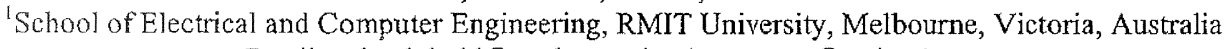 \\ Email: roland.dodd@student.rmit.edu.au,x.yu@rmit.edu.au \\ ${ }^{2}$ School of Computing Sciences, Central Queensland University \\ Rockhampton, Queensland, Australia. Email: a.chiou@cqu.edu.au \\ ${ }^{3}$ Queensland University of Technology \\ Brisbane, Australia. Email: r.broadfoot@qut.edu.au
}

\begin{abstract}
This paper discusses the framework of an expert advisory system designed to provide expert knowledge in the control and management of a sugar mill crystallization stage. The Smart Supervisory Control System (SSCS) is fundamentally a hybrid fuzzy logic based expert system that incorporates fuzzy logic, explanatory capabilities and dynamic interrelational process models of the crystallisation stage. The primary topic of this paper will be a description of the framework of the SSCS system with focus on: (1) modular design, (2) system layering, and (3) system features.
\end{abstract}

\section{INTRODUCTION}

Raw sugar production from cane is a nominally continuous operation, with 120-168 hours of processing per week, extending over 20-25 weeks of the harvest season. The crystallisation section, often loosely referred to as the pan stage, is the most complex part of the factory process where there are several batch wise or continuous crystallisation steps taking place concurrently [1].

In current Australian practice, two operators are normally employed on the pan stage and usually their duties extend no further than this section. There is considerable process interaction between the pan stage and centrifugal stage although management of the centrifugals is undertaken by different operators. The overall strategic management of the pan stage is quite difficult because of the very large number of process streams of varying compositions and crystal growth rate characteristics which must be managed [2]. Often the pan stage is managed in a sub-optimal manner because an overview of operations encompassing various sections - cane receival section, juice processing stations, the pan stage and centrifugal station - is not available.

The pressures on the Australian sugar industry to reduce the costs of sugar manufacture and increase the consistency of producing sugar of high quality require a smarter strategy for operation. This paper reports on the framework of a SSCS for sugar mill crystallization stage that has not been attempted in the world previously. Currently, there is no such supervisory control system for pan stage operations neither in the Australian sugar industry nor, as best as known to the collaborators, in the world sugar industry. The SSCS uses advanced intelligent technologies to provide a standardised approach for pan operations by integrating data from a variety of information sources from different sections of the sugar mill, along with dynamic process models of the pan stage and the collective knowledge and expertise of pan stage operators [3]. The integration of such features leads to a challenge in the design and development of the SSCS. Previous research [3] acknowledges that no conventional software engineering methods exist to provide a solution to this problem.

This paper is organised as follows. Section II discusses a system overview of the SSCS and explains how it fits into current sugar mill operations. Section III presents the basic framework used and the extensions required to the standard fuzzy logic based expert system approach. Section IV discusses the modular architecture of the application design with Section $V$ then presenting a discussion and conclusions.

\section{BACKGROUND}

The system specifications for key SSCS features require that the system recommendations and expert advice provide four core control strategies [4].

The four core control strategies for the primary system output are:

1. Pan duty management;

2. Pan control strategy;

3. Pan schedule management; and

4. Stock tank management.

Additionally, these core control strategies are supported by a further two secondary system outputs:

5. Prediction of future pan stage operating conditions for offered system recommendations compared to current operations; and

6. Explanatory and justification capabilities.

These secondary outputs are critical in providing reasoning and justifications for the recommended system advices. The system recommendations, prediction of future pan stage operating conditions and advice justifications that the pan stage operators receive from the SSCS will:

1. Result in a formal structure to the decision making procedure and reduce the number of ad hoc decisions and, consequently, the number of incorrect decisions; 
2. Achieve increased productivity with the existing equipment, by employing strategies that are recommended by the SSCS;

3. Make improved use of the equipment capabilities to achieve sugar recovery, sugar quality and steam consumption targets while fulfilling the production rate requirements; and

4. Forewarn of potential problems with the current operating strategies.

In order to achieve these objectives the SSCS uses the following information in its determination:

1. Projected cane crushing conditions and calculation of syrup input to the pan stage;

2. Projected production loading on the different pan stage operations;

3. Status of each pan, buffer storage tank and product receiver - accounting for the stage in production cycle or level of equipment;

4. Operating status of the centrifugal station;

5. Serviceability of all equipment items;

6. Mininum cycle times for each pan for current operating circumstances (for defined levels of yield and sugar quality); and

7. Steam consumption requirements for the individual pans to meet different target production rates.

The SSCS works in tandem with the pan stage operator and current pan stage computer control systems to perform the previously mentioned objectives and system advice. Fig. 1 provides a visual depiction of interactions between the current systems and the operator. Existing pan stage infrastructure consists of sugar mill control system that interfaces directly with, and controls the pan stage operations. A pan stage operator interacts directly with this system.

The SSCS is part of a cooperative control strategy and works in conjunction with the sugar mill control system and pan stage operators. It takes operator input along with information from the existing sugar mill control system via a real time parasitic data feed from the sugar mill control system databases. The SSCS influences pans stage processes through the actions performed by the operators. The operators use advices/recommendations, in line with the reasoning process

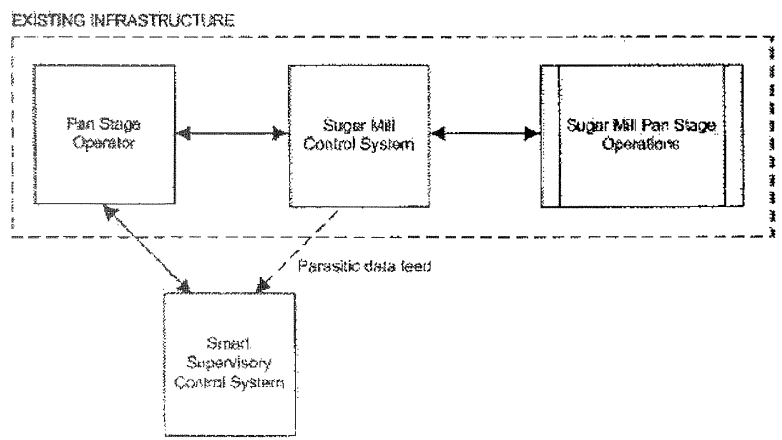

Fig. 1. Smart supervisory control system interaction diagram. from the SSCS, to influencing pan stage operations through their interaction with the sugar mill control system. Such an arrangement also serves to keep human decision making as part of the process.

\section{OVERVIEW OF THE FRAMEWORK}

The SSCS is essentially a hybrid fuzzy logic based expert system incorporating fuzzy logic, explanatory capabilities and industrial process models of the pan stage. The knowledge base is composed of human operator knowledge coupled with mathematic dynamic models describing the crystallization process. The integration of such features leads to a challenge in the design and development of the SSCS. Previous research [3] acknowledges that no conventional software engineering methods exist to provide a solution to this problem.

\section{A. Modular Architecture}

The SSCSs modular architecture is based upon conventional expert systems $[5,6]$ and conventional If-Then fuzzy rule based systems design $[7,8]$.

As depicted in Fig. 2 the SSCS is designed as a modular architecture with clustered elements performing layered tasks. The partitioning of the system architecture aids in maintenance, accountability, upgradeability, adaptability and flexibility [9].

Fig. 2 provides a simplified representation of the overall system design. This representation gives a clear comparison to the "standard" fuzzy logic expert system framework that is commonly used and highlights the extensions that have been engineered.

Compared to the conventional fuzzy logic expert system design, the editor layer is essentially unchanged with only the addition of an editor to customize parameters of the industrial process models of the pan stage. The data layer now also includes the dynamic interrelation models of the crystallisation stage. The defuzzification component that is typical of fuzzy logic expert systems has been replaced by a meta-consequent function [10]. The support and real-world layers have also been

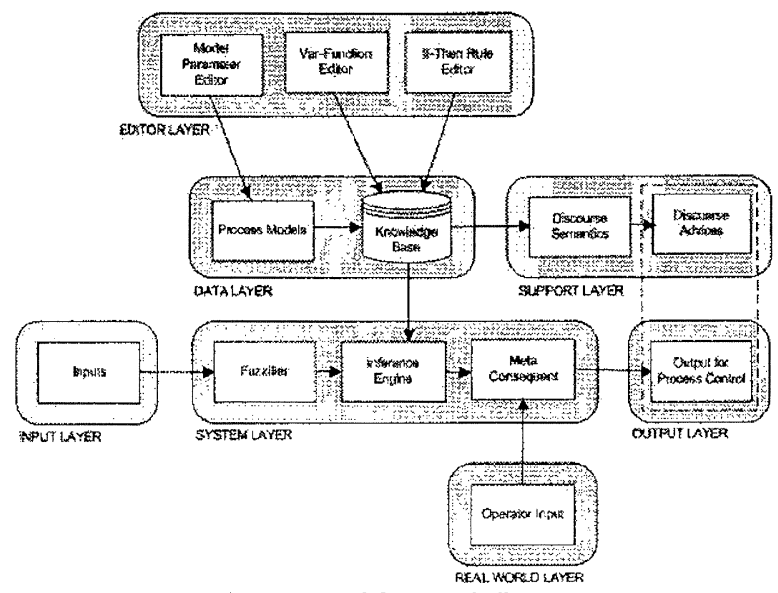

Fig. 2. Layered framework diagram 
added. The input and output layers have also been separated. The industrial process models of the pan stage are now tightly integrated with the expert system rule base and work in tandem.

\section{B. Loyering}

This unique modularity gives rise to the following modified layers:

- Editor Layer - Fuzzy variable function editor, If-Then rule editor and dynamic pan stage process model parameter editor.

- Data Layer - Knowledge base and process model components.

- System Layer - Fuzzifier, inference engine and metaconsequent components.

- Input layer - Parasitic data link to sugar mill control system information sources.

- Output layer - Discourse advices and output for process control components.

- Support layer - Discourse semantics and discourse explanatory components.

- Real world layer - Data from external information sources via user interface component.

\section{MODULAR ARCHITECTURE}

Fig. 3 displays the modular architecture of the application design. This application design follows the framework presented in Fig. 2. The design architecture is modular with clustered elements performing layered tasks. This architecture is based upon [11] but with extension in order to merge the dynamic interrelational process models of the pan stage with the fuzzy rule base. Associated changes also include an innovative blackboard system for information storage of process model results predicting future pan stage operating conditions, databases for parameter storage of the dynamic interrelational pan stage models and editing facilities for parameter tuning of the dynamic pan stage process models.

\section{A. Input Layer}

The input layer draws its information directly from the sugar mill online control system having been published as a series of relational databases. This information provides current real time factory data on the cane receival sections, juice processing station, the pan stage and centrifugal station. Further information from the crystallisation stage operators, through the graphical user interface, can be provided to assist in determination of equipment performance ratings and operational problems and characteristics of the syrup, molasses and sugar process streams along with crushing season information.

\section{B. Editor Loyer}

The editor layer provides the knowledge engineer with the capabilities to modify the fuzzy membership function parameters and rule associations with the dynamic process models. This layer also provides the facilities to tune the dynamic pan stage process model parameters that are associated with the rule base and also to assign or modify the explanations tagged to each of the rules.

\section{Data Layer}

The data layer consists of a series of databases for information storage. All databases use a standard RDBMS for interoperability and system interfacing. The final membership functions and fuzzy If-Then rule base are stored in the knowledge base at the data layer along with parameters specific to the industrial pan stage models and discourse knowledge bases. The data layer also includes an innovative blackboard system that acts as a dynamic storage repository for results from the dynamic interrelational models of the pan stage. The blackboard system stores all major results for each future time interval prediction of process model variables. Sugar mill control system data containing information from cane receival, juice processing station, the pan stage and the centrifugal station is also stored. Dynamic user input from the pan stage operator is also captured for later use.

\section{System Layer}

The SSCS utilizes four key subsystems to carry out data processing of the two major sources of system input. These are transformed into the six required system outputs. The system layer is the most complex of the layers in the SSCS architecture and essentially comprising the majority of system software operations. These four innovative modules utilize multithreading for each subsystem. Each module runs as part of a separate thread to ensure system execution will continue even under the event of a subsystem failure. Furthermore these modules feature extensive use of exception handing for failure prevention and each is based, as is the rest of the software development, on object oriented design. These core subsystems as shown in Fig. 3 are:

1. Dynamic interrelational process models of the pan stage;

2. Inference engine delivering fuzzy meta results;

3. Prediction of future pan stage operating conditions; and

4. Explanatory and justification facilities

The process models developed to describe the dynamic features of the pan stage process and forming one of the key subsystems in this layer are:

1) Pan and fugal phase determination models. This model determines the status and phase of schedule that vacuum pan and centrifugal station is currently at. The current operating status of the vacuum pans can be determined using a simplified classification procedure based upon steam usage, level and change in level as presented in [12]. Receiver levels allow fugal station status to be inferred. Once this determination is made this model is coupled with the pan stage schedule and the further described process models for prediction of future pan stage operating conditions.

2) Syrup prediction model. This model determines the 


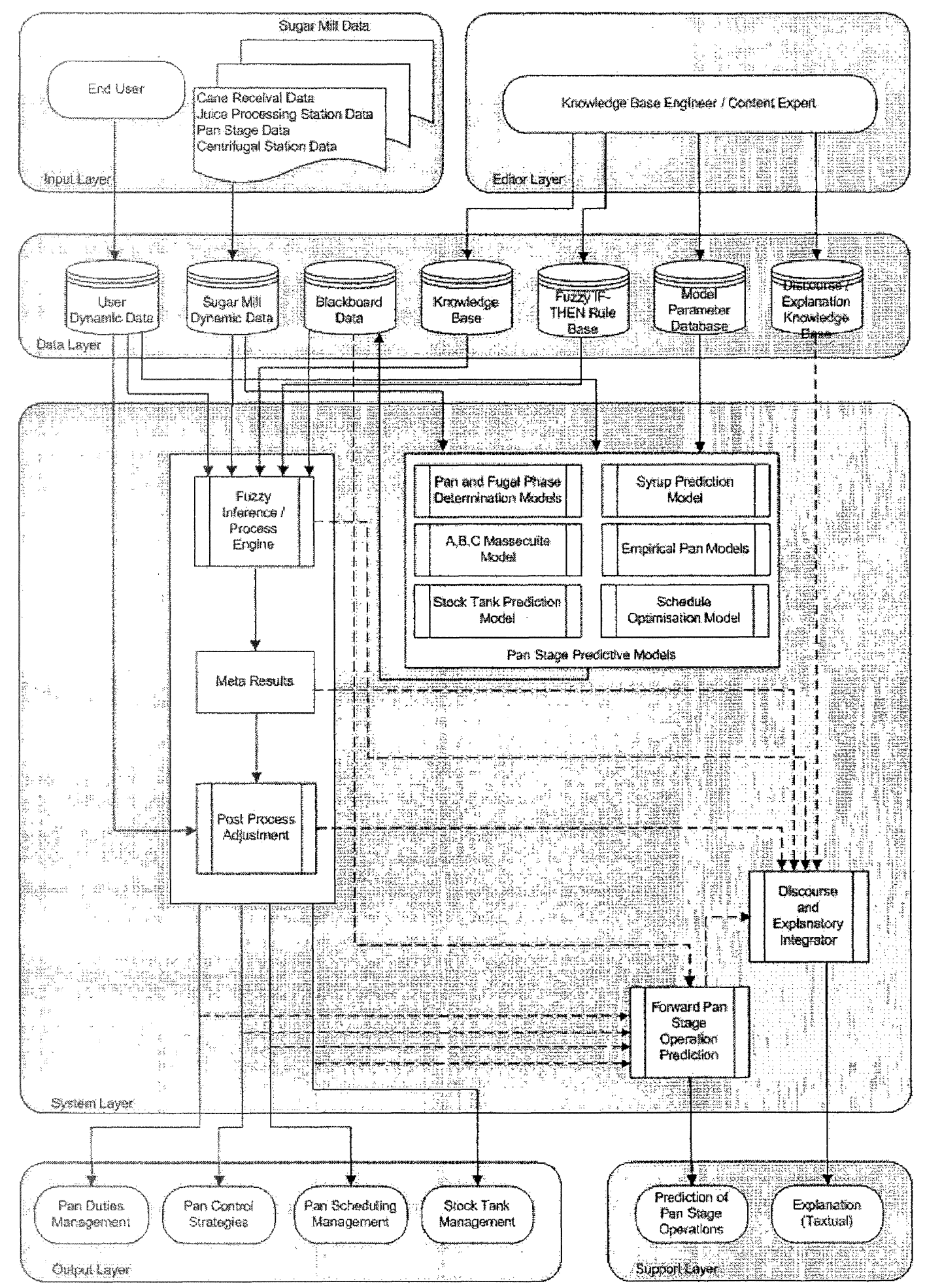

Fig, 3. Detailed application design diagram. Dashed lines indicate that the supporting subsystem modules, providing the secondary system outputs, do not influence primary system outputs. 
future flow of syrup to the pan stage for bins of cane entering the factory after the harvesting process. The prediction of the quantities of sucrose and impurities in syrup allows a forward forecasting of the future pan stage loading of syrup [13]. The syrup prediction model uses cane receival data combined with juice processing station information and pan stage operator knowledge to forward predict syrup quantities and composition. This model is of key importance as syrup comprises the basic input to the pan stage.

3) A, B, C Massecuite model. This steady-state model of the pan stage has been developed to calculate average flow rates of process streams using mass balances at each vacuum pan, stock tank and fugal [13]. The model determines the average production rates of vacuum pan massecuite, $\mathrm{C}$ sugar remelt, molasses and sugar streams given the syrup purity and flow rate to the pan stage. Crystal sizing determinations assist in calculating the necessary quantities of $\mathrm{C}$ sugar needed for the $\mathrm{A}$ and $\mathrm{B}$ pans to ensure final product sugar is of the required size. Typical conditions for crystal content, sugar size and coefficient of size variation have been assumed in this model and the purity rise of sugar at the fugals has also been taken into account.

4) Empirical vacuum pan models. Individual pan production rates have been modeled by constructing empirical relationships [14] for the rate at which each pan takes feed material (liquor, A molasses or B molasses) during the different phases of the pan's operation. The current operating phase being determined by the pan phase determination models described previously. This boil-on rate for feed materials is a function of the massecuite level and phase of the pan, steam rate, head space pressure (vacuum), brix and purity of the feed liquor/molasses. Using this method it is possible to construct a piece-wise model of pan feed rate characteristics during each phase of the pan's operation for each of the vacuum pans.

5) Stock tank prediction models. With the empirical model for each vacuum pan having been established by the empirical pan models, the boil-on rates for each feed stream at the different stages of the pan stage schedule can be determined by summing the liquor, A molasses and B molasses feed rates for all the pans at that point in the schedule. Given the expected liquor production rate and $\mathrm{C}$ sugar remelt production rate to the liquor tank during this interval, the predicted tank levels can be determined for the liquor tank. Similarly the predicted tank levels for the $A$ and $B$ molasses streams can be calculated from the production rates of the molasses at the centrifugal station and the sum of the consumption rates on the individual pans at a specific point in the pan stage schedule.

6) Schedule optimization model. Using the previously established models in conjunction with genetic algorithm optimization, the scheduling of when pans should start and complete strikes can be made in order to avoid vacuum pan idling time, while minimizing steam usage on the overall pan stage and adhering to sugar production productivity, recovery and quality requirements.

This information in conjunction with other information sources gathered through the input layer work collectively with the inference process and process models to provide information on:

- Recommended steam rate usage for the vacuums pans phase given current operating conditions;

- Choice of A/B massecuite duties for "swing" vacuum pans;

- Forecasting of stock tank levels and future disturbances;

- Footing quantities to the vacuum pans; and

- Scheduling when pans should start and complete strikes.

The fuzzy inference engine is another core subsystem in the system layer. The meta-consequent component in this subsystem replaces the defuzzier found in traditional fuzzy logic based expert systems and instead of providing defuzzified results it produces meta-results through the use of fuzzy metaconsequent functionality [10]. The meta-consequent functionality provides an adjustment to the results of the inference process and modifies the dynamic pan stage process models output to correlate with information provided by the pan stage operators through the input layer.

This subsystem is complimented by the real world input from the input layer to provide a method for mapping information on equipment performance ratings and operational problems and characteristics of the syrup, molasses and sugar process streams to the dynamic process models.

This provides meaningful interpretation of the inference process output by matching it to the operating conditions at the crystallisation stage.

The inference engine subsystem involving meta-consequent functions works in tandem with dynamic interrelational process models of the pan stage to determine the primary system output control strategies.

These outputs are further justified by the explanatory subsystem which provides system explanation of final control strategies. Previous research [9] has show the need for expert system recommendations to be further accompanied by explanations to aid in the understanding and justification of presented advices to gain user acceptance. Limited research into explanatory capabilities for fuzzy expert systems has been carried out [15]. In recent times some innovation has been performed [16].

Explanations tagged to each rule in the If-Then fuzzy rule base are triggered and propagate through the inference mechanism upon a rule firing. This information is further passed to the support layer for further processing.

A prediction subsystem is also provided as secondary system output to support the primary control strategies. This subsystem provides a prediction of future pan stage operating conditions for offered system recommendations compared to 
current operations. A forecast of current operations is made against operations utilizing the system recommended control strategies.

\section{E. Output Layer and Support Layer}

On the completion of the system layer processes the final results are passed to the output and support layers. The four primary control strategy recommendations are passed to the output layer for formatting. The secondary supporting results are passed to the support layer for formatting and display.

The support layer works to consolidate the inference process by formatting justifications for the presented advice in the most appropriate format. The method of presentation is an integral part of the output layer. The justification process is independent of the inference process which provides the final control output values that are recommended.

Aside from English based textual justification, justification for recommendation can be provided as a series of graphs or measures to show previous data trends on the pan stage schedule, productivity and steam rate usage. These can be presented against forward predictions of current pan stage operations and depict bow the system recommendations can provide improvements.

\section{DISCUSSION AND CONCLUSIONS}

The overall strategic management of the pan stage is quite difficult because of the very large number of process streams of varying compositions and crystal growth rate characteristics which must be managed. Often the pan stage is managed in a sub-optimal manner because an overview of operations encompassing various sections cane receival sections, juice processing stations, the pan stage and centrifugal station - is not available.

This paper reports a work in progress in the development of a SSCS for sugar mill crystallization stage support. The SSCS framework presented is an innovative architecture to unify fragmented systems of data from pan stage operators, fuzzy rule base, developed industrial process models of the pan stage and information sources across varying sections of the sugar mill to solve an important industrial control problem. An innovative modular system architecture based upon a layered system framework has been presented along with major system features.

This system seeks to provide a unifying structure to assist pan stage operators in making early decisions, such as changes to steam rates, or allocation of pans to different duties to avoid production rate difficulties, and maintain good operational performance with respect to sugar quality, sugat recovery and minimization of steam consumption on the total pan stage.

The SSCS is a hybrid fuzzy expert system incorporating fuzzy logic, explanatory capabilities and industrial process models of the crystallisation stage. Presently the most difficult part of process modelling has been completed and the construction of the advisory system is being undertaken.

\section{ACKNOWLEDGMENTS}

The project contributors would like to thank the staff at Racecourse Sugar Mill, Mackay, Australia for their cooperation and support. This project is supported by an Australian Research Council linkage grant. The funding assistance provided by the Sugar Research Institute, Brisbane, Australia is also acknowledged and appreciated.

\section{REFERENCES}

[1] R. Broadfoot and A. Beath, (1998). "Modelling of batch sugar centrifugal performance", in Proceedings of 26th Australasian Chemical Engineering Conference, 1988, p.294-303

[2] K. Miller and R. Broadfoot. "Crystal growth rates in high grade massecuite boilings", in Proceedings of the Australian Society of Sugar Cane Technologists Conference, (1997), p.441-447

[3] R. Dodd, X. Yu, R. Broadfoot and A. Chiou, (2002). "Development of a smart supervisory control system for pan stage operations in sugar factories", in Proceedings of Information Technology in Regional Areas, 2002, Central Queensland University, Rockhampton, Australia, p.368375.

[4] X. Yu and R. Broadfoot, (2001). "Developing a Smart Supervisory Control System for Pan Stage Operations in Sugar Factories"; Australian Research Council linkage project application number LP0230710.

[5] K. Leung and M. Wong, "An Expert-System Shell using Structured Knowledge: an Object Oriented Approach", Computer, (1990), 23(3), 38 46

[6] A. Gisolfi and W. Balzano, Constructing and consulting the knowledge base of an expert systems shell. Expert systems. (1993), 10(1), 27-37

[7] S. Goel, K. Modi, M. Shrivastava, P. Chande and A. Gaiwak, (1995). "Design of a Fuzzy Expert System Development Shell", in Proceedings of 1995 IEEE Anmual International Engineering Management Conference, 1995, p.343-346

[8] R. Berkan and S Trubatch, Fuzzy Systems Design Principles: Building Fuzzy If-Then Rule Bases. IEEE Press, 1997

[9] L. Ye and P. Johnson, "The Impact of Explanation Facilities on User Acceptance of Expert System Advice", Expert Systems with Applications (1995), 9(4), 543-556

[10] A. Chiou A and X. Yu, (2007). "Remote Sensing in Decision Support Systems: Using Fuzzy post Adjustment in Localisation of Weed Prediction", in Proceedings of 2007 International Conference on Intelligent Sensors, Sensor Networks and Information Processing, 2007, Melbourne, Australia.

[11] A. Chiou A and X. Yu, (2007). "A Large-Scale Agro Decision Support System: Framework for (Physical) Fusion of a Multi-Input and MultiOutput Hybrid System", in Proceedings of 2007 International Conference on Intelligent Sensors, Sensor Networks and Information Processing, 2007, Melbourne, Australia.

[12] J. Frew and P. Wright, "Pan Stage Advisory Scheme at Racecourse Mill", Commonwealth Scientific and Industrial Research Organization Australia, 1977, report number CE/R-52.

[13] R. Dodd, R. Broadfoot, X. Yu and A. Chiou, (2005). "Development of Smart Supervisory Control System in a Sugar Mill Crystallisation Stage", in Proceedings of I*PROMS Virtual Ynternational Conference, 2005, p.527-534

[14] R. Dodd, R. Broadfoot, X. Yu and A. Chiou, (2005). "Empirical Pan Modelling of Vacuum Pans for a Sugar Mill Crystallization Stage", in Proceedings of Australian Society of Sugar Cane Technologists, 2005, Bundaberg, Australia, p.423-436

[15] S. Gregor and X. Yu. Exploring the Explanatory Capabilities of Intelligent System Technologies. Second International Discourse with Fuzzy Logic in the New Millennium. Physica-Verlag. 2000, pp 289-300.

[16] A. Chiou A and X. Yu, (2007). "Industrial Decision Support System (IDSS) in Weed Control and Management Strategies: Expert Advice Using Descriptive Schemata and Explanatory Capabilities", in Proceedings of The $33^{\text {rd }}$ Annual Conference of the IEEE Industrial Electronics Society, 2007, Taipei, Taiwan, p.105-110 